\title{
External Audit System of Project Evaluation: Optimal Structure and Audit Quality
}

\author{
Jun-ya FUKUMOTO \\ Department of Environmental Studies, Graduate School of Frontier Sciences, \\ The University of Tokyo, 7-3-1 Hongo, Bunkyo-ku, Tokyo 113-8654, Japan \\ E-mail: fukumoto@k.u-tokyo.ac.jp
}

Received November 16, 2004; final version accepted February 7, 2005

\begin{abstract}
It is pertinent to build an effective external audit system to prevent manipulations in the analysis of project evaluation. In reality, it is difficult for an external auditor to understand details of the analysis. Hence, it is likely that he would misinterpret and penalize the analysts even though they have not manipulated the analysis. The possibility of misinterpretation gives analysts the disincentive for participating in project evaluation business. In the long term, this would hinder the implementation of socially desirable projects. This paper tries to derive the optimal structure of external audit system of project evaluation focusing on the quality of audit. We modify and analyze the principal=agent=auditor model which we suggested in Fukumoto and Tsuchiya (2004).
\end{abstract}

KEYWORDS: project evaluation, manipulation, external audit system, quality of audit

\section{Introduction}

Since 1990's, the inefficiency of public investment projects has been severely criticized by the general public in Japan. Many insisted that the introduction and implementation of project evaluation system was necessary. Correspondingly in 1998, the Japanese government decided to introduce an evaluation system to improve the efficiency and transparency of public investment projects.

However, many say that public investment projects are still inefficient though the project evaluation system has been introduced. They state that the public investment related government ministries are merely seeking ways and means to justify the implementation of projects that maximize their budget and are manipulating the analysis of project evaluation. They raise their doubts on the legitimacy and effectiveness of current project evaluation system. In the long run, the lack of social reliability on the project evaluation system would thwart the implementation of socially desirable projects. To address this issue, we need another supporting system that prevents manipulations in the analysis of project evaluation. This is the audit system of project evaluation. With this system, the analysts of project evaluation are penalized if an auditor finds manipulations.

In essence, there are two typical audit systems (Williamson, 1985; Kofman and Lawarree, 1993). First, the internal audit system and the other is the external audit system. In the former, auditors (internal auditors) belong to the same organization with auditees. Internal auditors possess abundant information on the activity of the auditees, which is not limited to the scope of auditing. Since they can provide both the auditing and non-auditing services, the opportunity cost of the auditing function is low. In contrast, the latter sees the auditors (external auditor) belonging to a different organization from auditees. They have less information on the activity of auditees and the opportunity cost of the auditing function is high. The internal audit system is superior to the external one from the viewpoint of cost of auditing. However, the risk of collusion is relatively higher in the internal audit system compared with the external one. This is because internal auditors are likely to suffer pressures in hiding and covering unfavorable results from the audit in favor of their organization. In the external audit system, in which the external auditors and auditees are independent of one another, external auditors can disregard such pressures. Therefore, to ascertain which audit system is superior depends on the characteristics and surrounding environment of the audit.

In the case of the audit system of project evaluation, the most important factor is the social reliability on the project evaluation system. Even if no evidence of collusion were found, the mere possibility of its occurrence would reduce social reliability drastically. Therefore, as for the audit system of project evaluation, we are of the opinion that the external one is more effective. However, this brings us to the fact that the analysis of project evaluation is difficult for anyone other than the analyst to understand. There is no guarantee that external auditors can perform accurate auditing. External auditors may point out manipulations owing to misinterpretation and the analysts take the penalty unjustly. The probability of misinterpretation gives the analyst disincentives for participating in project evaluation business. In the long run, it hinders the project evaluation system to function effectively. Therefore, when we search for the optimal structure of external audit system, we must take full account of the possibility of misinterpretation by external auditors.

This paper tries to derive the optimal structure of external audit system of project evaluation focusing on the probability of misinterpretation by external auditors. We modify and analyze the principal=agent=auditor model 
which we suggested in our precedent research (Fukumoto and Tsuchiya, 2004). Based on the model analysis, we will demonstrate that the probability of misinterpretation makes it difficult to prevent manipulations in the analysis of project evaluation. Furthermore, we will demonstrate how the optimal structure depends on the accuracy and economical efficiency of external auditors.

\section{Basic Framework}

\subsection{Current system of project evaluation}

In 1998, all the public investment related ministries decided to introduce the project evaluation system. This system consists of three systems. First is the ex-ante evaluation system. With the first system, ex-ante evaluation is performed for all the projects at the planning stage to judge whether they should be adopted and budgeted. Next is the revaluation system. With this system, ex-ante evaluation or revaluation is performed for all the ongoing projects that satisfy specific conditions to judge whether they should be continued or suspended. The other is the ex-post evaluation system. With this system, ex-post evaluation is performed for some projects to accumulate beneficial knowledge in planning and implementing new projects. To date, the ex-ante evaluation and the revaluation system have been working. Especially as for the ex-ante evaluation system, the ministries have created the manual of evaluation for each infrastructure facility, and ex-ante evaluation has been done for all the public investment projects.

The ministries order consultants to perform project evaluation and to report its result. The analysts of consultants perform project evaluation based on the official manual of project evaluation. The manual specifies the methodology and the detailed procedure of project evaluation. However, the analysts must consider and weigh various factors needed for the analysis. The examples of such factors are socio-economic model, data and basic unit parameters. As for the selection of these factors, there is no unified rule in spite of the research development for the past few decades. The analysts can select many factors at their own discretion. This flexibility makes it easy for the analysts to manipulate the analysis of project evaluation. Since it is believed that Japanese government ministries that give out orders have strong power over the consultants that accept orders, many suspect that the government ministries may be making the analysts to manipulate.

The important roles of the audit system of project evaluation are to give advises for the improvement of the quality of project evaluation and to prevent the manipulation of the analysis of project evaluation. Japanese government ministries were re-organized in 2001. In this re-organization, the audit system of project evaluation was also reformed. Key changes are as follows. 1) Each public investment related ministry established a new section which plays the role of auditors of project evaluation completed by the other sections. 2) Administrative Evaluation Bureau (AEB) in Ministry of Internal Affairs and Communications, which evaluates the policy evaluation process in each government ministries, was formed. 3) Committee for Policy Evaluation and Independent Administrative Agency Evaluation (CPEIAAE), which plays the role of auditors and advisors of AEB, was created.

In the above-mentioned system, what should be noted is that each public investment related ministry does not only perform project evaluation but also audits. This is so intended that the abundant knowledge about public investment projects be utilized efficiently. However, this system has a severe weakness from the viewpoint of social reliability. Since this audit system corresponds to the internal audit, the general public cannot remove their doubts on the collusion between the auditor and the auditee. AEB and CPEIAAE are prepared to prevent such collusions by evaluating the whole process of project evaluation. However, we are of the opinion that it is difficult for AEB and CPEIAAE to prevent collusion among administrative unit that belong to the same organization. We cannot help but think that the current audit system of project evaluation is not effective to recover the social reliability on the project evaluation system.

\subsection{Overview of the principal=agent=auditor model}

In the agency theory (see for example, Laffont and Tirole, 1993; Salanie, 1997; Macho-Standler and Perez-Castrillo, 2001), many attempts have been made to search for the optimal audit system. We are of the opinion that it is effective to approach the above mentioned problem following this theory. The most fundamental approach of the agency theory is called "Revelation Principle" (see for example, Myerson, 1982). This principle made it easy to characterize the mechanism design problem under asymmetric information and to conduct normative analysis. However, it is not without limitations. The revelation principle can not be applied easily to the mechanism design problem of organizations where the possibility of collusion exists. One of the most seminal papers that considered collusions in organizations is Tirole (1986). He suggested the principal=agent=auditor model which consists of three players (usually known as principal=agent=supervisor model). In this model, an auditor monitors the activities of an agent to prevent his negligence of duty. However, even if an auditor finds the agent's negligence, there is the possibility of collusion between the auditor and the agent. Therefore, the means to prevent collusion is the most essential point in Tirole's paper. The most striking result in his paper is that the optimal contract is collusion-proof, that is, when we seek to formulate the optimal contract, we do not heed for contracts that cause collusion between an auditor and an agent. This makes it easier for us to seek for the optimal contract. In reality, however, there are many cases of collusion and it 
seems to be almost impossible to remove all the collusions. Post Tirole's paper, many attempts have been made to search for the conditions under which collusions happen even in the optimal system (for example, Tirole, 1992, Itoh, 1993; Che, 1995; Kofman and Lawarree, 1996; Strausz, 1997; Laffont and Martimort, 1997).

It is clear that the collusion between an auditor and an agent happens only when both players possesses interests at stake from it. Collusion does not happen when the total interests of both players do not increase by colluding. It does not happen either when there is no means to transfer their interests from one to the other, even if the total interests of both players increase by colluding. If an auditor and an agent belong to different organizations, that is, if the audit system is an external one, the conditions of collusion are not likely to be satisfied. As we have mentioned in the Introduction, the most important purpose of introducing the audit system of project evaluation is to improve the social reliability on the project evaluation system. We are of the opinion that the external audit system is more effective than the internal one to recover the social reliability since the mere possibility of collusion would reduce social reliability drastically. Therefore, in the following analysis, we focus our discussion on the optimal external audit system and analyze the principal $=$ agent $=$ external audit model of project evaluation.

Before going into the discussion on the optimal external audit system of project evaluation, we had better mention the approaches to address the issue other than the introduction of the audit system of project evaluation. It is believed that the government ministries have incentives to increase their budget and to justify the implementation of their projects. This is the fundamental reason of the lack of social reliability on the project evaluation system. Therefore, the simplest way to tackle with the issue is to reduce the incentive of the government ministries to increase their budget. The reformation of the administration system, the reinforcement of politicians' ability of monitoring the government agencies and the introduction of PFI system into infrastructure development are the examples of such approaches. We can analyze the impact of these approaches based on the agency theory (see for example, Tirole, 1994; Laffont and Martimort, 1999; Laffont, 2000; Persson and Tabellini, 2000). However, this brings us to the fact that we must consider the more fundamental structure of the government organization. We are of the opinion that the introduction of the external audit system is the not only effective but also realistic way of dealing with the issue. In this paper, we concentrate our discussion on the external audit system. We want to pick up other approaches in another paper.

\subsection{Principal=agent $=$ audit model of project evaluation}

The flexibility left for the analysts of project evaluation makes it easy for the government agencies to manipulate the analysis of project evaluation and to justify the implementation of their projects. When we search for the optimal audit system of project evaluation, we must be mindful of the flexibility left to the analysts.

Fukumoto and Tsuchiya (2004) is the first ever paper that explored the optimal audit system of project evaluation based on the agency theory focusing on the discretion to be left to the analysts. They assumed that the analyst can use two models for project evaluation and select one of the results to be reported. The plurality of "model" in their analysis expresses not only the diversity of approaches for project evaluation (such as general equilibrium approach and hedonic approach) but also the difference of functional forms (such as linear function and log-linear function), the difference of data used to estimate parameters or the difference of explanatory variables used in the analysis. In reality, the analysts of project evaluation calculate the economic efficiency of projects for many patterns of functional forms, data and explanatory variables, and report only one or two result of such calculation to the government ministries. Fukumoto and Tsuchiya described such situation in their model.

They suggested the principal=agent=auditor model of project evaluation. In their model, the auditor reviews the reported result and the whole process of project evaluation, and reports to the principal whether the agent has reported the right result of the analysis. They analyzed both the cases of internal and external audit system and derived the optimal structure of audit system of project evaluation that is dependent on the benefits of projects and the cost of auditing. Furthermore, they showed the complementary relationship between the introduction of audit system and the creation of the manual. Their analysis should be valued for its novelty and timeliness. However, it is not without limitations. The first would be that individual rationality condition of the analysts was not taken into account. The probability of misinterpretation by the external auditors was not considered. The misinterpretation by the external auditors consists of two types: type I error and type II error. In the former, even if the analyst has not manipulated, the external auditor misinterprets that the analysts has manipulated the analysis of project evaluation. In the latter, even if analysts have manipulated, an external auditor cannot find proof of manipulation. Fukumoto and Tsuchiya considered only towards type II error. As mentioned in the Introduction, the high possibility of type I error offers disincentives to the analysts for participating in project evaluation business. In this paper, we will tackle this problem by modifying the analysis of Fukumoto and Tsuchiya to consider the possibility of type I error and the individual rationality condition of the analysts.

\section{The Model}

\subsection{Premises of the model}

Our model consists of three players, that is, a principal, an agent and an external auditor. The principal is the citizen. 
Table 1. Accuracy of project evaluation model.

\begin{tabular}{|c|c|c|}
\hline \multicolumn{3}{|c|}{ Case 1 (probability $p_{1}$ ) } \\
\hline & $m_{1}$ (correct) & $m_{2}($ correct $)$ \\
\hline$a_{1}$ & $B_{1}$ & $B_{1}$ \\
\hline$a_{2}$ & $B_{2}$ & $B_{2}$ \\
\hline \multicolumn{3}{|c|}{ Case 3 (probability $p_{3}$ ) } \\
\hline & $m_{1}$ (wrong) & $m_{2}($ correct $)$ \\
\hline$a_{1}$ & $B_{2}$ & $B_{1}$ \\
\hline$a_{2}$ & $B_{1}$ & $B_{2}$ \\
\hline
\end{tabular}

\begin{tabular}{|c|c|c|}
\hline \multicolumn{3}{|c|}{ Case 2 (probability $p_{2}$ ) } \\
\hline & $m_{1}($ correct $)$ & $m_{2}$ (wrong) \\
\hline$a_{1}$ & $B_{1}$ & $B_{2}$ \\
\hline$a_{2}$ & $B_{2}$ & $B_{1}$ \\
\hline \multicolumn{3}{|c|}{ Case 4 (probability $p_{4}$ ) } \\
\hline & $m_{1}$ (wrong) & $m_{2}$ (wrong) \\
\hline$a_{1}$ & $B_{2}$ & $B_{2}$ \\
\hline$a_{2}$ & $B_{1}$ & $B_{1}$ \\
\hline
\end{tabular}

They enjoy public facilities provided through public investment projects. The agent is the public investment related government ministry. He is commissioned by the principal to plan public investment projects, to perform project evaluation and to implement public investment projects. The external auditor makes the inference on the analysis of project evaluation done by the agent. The objective of auditing is to prevent manipulations by the agent. As an example of the external auditor, we consider the official auditing institution such as AEB and CPEIAAE.

We assume the following simplified situation. There are two alternatives of public investment projects. We denote these as $a_{i} \in\left\{a_{1}, a_{2}\right\}$. Only one of the two alternatives will be implemented. The net benefit (afterward NB) of the principal that happens when the alternative $i$ is implemented is $B_{i} \in\left\{B_{1}, B_{2}\right\}$ where $\Delta B \equiv B_{1}-B_{2}>0$. The private interest of the agent that happens when the alternative $i$ is implemented is $R_{i} \in\left\{R_{1}, R_{2}\right\}$ where $\Delta R \equiv R_{2}-R_{1}>0$. Though the NB of alternative 2 is lower than that of alternative 1, the agent wants to implement alternative 2, the private interest of which is higher than that of alternative 1. The budget maximizing administrative organization described in Niskanen (1994) is assumed and this is the reason why the order of the NB and that of the private interest are different. The principal knows that the $\mathrm{NB}$ and the agent's private interest of one alternative are $\left(B_{1}, R_{1}\right)$ and those of the other one are $\left(B_{2}, R_{2}\right)$. But, they do not know whether those of alternative 1 are $\left(B_{1}, R_{1}\right)$ or $\left(B_{2}, R_{2}\right)$. On the other hand, the agent who has got immense experiences in public investment projects has perfect information on the above relationship. The agent can use this information asymmetry for their pursuit of private interest. In contrast, the principal obliges the agent to evaluate the economic efficiency of each alternative and to report the results. The principal tries to control the opportunistic behavior of the agent and to make the agent to implement the most desirable alternative for the principal.

There are two models used for project evaluation. We denote these as $m_{i} \in\left\{m_{1}, m_{2}\right\}$. The plurality of the models expresses not only the diversity of approaches for project evaluation but also the difference of basic unit parameters, functional forms, data or explanatory variables. As we have already mentioned in Section 2.3, this assumption corresponds with the reality of project evaluation. As known, every model of project evaluation that is used in the real world has its own advantages and disadvantages and sometimes leads to the wrong conclusion. So, we assume as follows that both two models calculate wrong values of NB with positive probabilities (see Table 1). With probability $p_{1}$, both two models calculate the correct values of $\mathrm{NB}$, that is, the NB of alternative 1 is $B_{1}$ and that of alternative 2 is $B_{2}$ (case 1). With probability $p_{2}$, model 1 calculates the correct values, that is, the NB of alternative 1 is $B_{1}$ and that of alternative 2 is $B_{2}$, but model 2 calculates the wrong values, that is, the NB of alternative 1 is $B_{2}$ and that of alternative 2 is $B_{1}$ (case 2). With probability $p_{3}$, model 1 calculates the wrong values, that is, the NB of alternative 1 is $B_{2}$ and that of alternative 2 is $B_{1}$, but model 2 calculates the correct values, that is, the NB of alternative 1 is $B_{1}$ and that of alternative 2 is $B_{2}$. With probability $p_{4}$, both models calculate the wrong values of NB, that is, the NB of alternative 1 is $B_{2}$ and that of alternative 2 is $B_{1}$. The knowledge about the accuracy of each model increases in academic societies, as the case studies of project evaluation accumulate. Therefore, we assume that four probabilities $\left(p_{1}, p_{2}, p_{3}, p_{4}\right)$ are common knowledge of the principal and the agent.

In case 1 , the NB of alternative 1 is evaluated higher than that of alternative 2 regardless of the model used and is always implemented. In case 4 , in a similar way, alternative 2 is always implemented. Case 1 and case 4 do not lead to a discussion. On the other hand, case 2 and case 3 lead to a discussion. In case 2, alternative 1 is implemented if the agent reports only the result of model 1 and alternative 2 is implemented if the agent reports only the result of model 2 . By selecting the result of model analysis to be reported to the principal, the self-interested agent can make alternative 2, from which he receives higher private interests and the principal receives lower NB than from alternative 1 , to be implemented. In a similar way, in case 3, by reporting only the result of model 1, the agent can make alternative 2 to be implemented. These behaviors are rational for the agent, but decrease the principal's NB. In this paper, we define the agent's opportunistic model selection as the manipulation of the analysis of project evaluation. As for the result of project evaluation, we assume that it is hard information, that is, it can not be falsified but can be hidden from the principal and the external auditor. Since the analysis of project evaluation is very complicated, it is difficult for anyone other than the analyst to check whether it is not falsified. Therefore, it seems to be more natural to assume that the result of project evaluation is not hard information but soft information. However, it is risky for the analysts to falsify the 
result since they are sometimes requested to submit not only the result of analysis but also the data or program used in the analysis. Furthermore, they can manipulate the result by selecting results to be reported even if they do not falsify the result. Therefore, we are of opinion that the assumption of hard information is appropriate.

The main role of the external auditor is to prevent the manipulation by the agent. He makes the inference on the analysis of project evaluation that was reported to the principal and judges whether the agent had indeed manipulated the analysis or otherwise. In case 1 and case 4 where the agent has no incentive to manipulate, the external auditor makes the correct inference. However, in case 2 and case 3 where the agent has the incentive to manipulate, the external auditor cannot always find the manipulation even if the agent has manipulated. Furthermore, in these cases, the external auditor misinterprets that the agent has manipulated with some positive probability, even though the agent has not manipulated. We denote the probability, with which the external auditor finds the manipulation when the agent has manipulated in case 2 and case 3 , as $0 \leq q_{1} \leq 1$, and denote the probability, with which the external auditor misinterprets that the agent has manipulated in case 2 and case 3 , as $0 \leq q_{2} \leq \bar{q}_{2}$ where $0 \leq \bar{q}_{2} \leq 1$ is a constant. Consequently, if the agent has manipulated, the auditor judges correctly with probability $q_{1}+\left(1-q_{1}\right) q_{2}$, and if the agent has not manipulated, the agent judges correctly with probability $1-q_{2}$. In this case, the probability of type I error is $q_{2}$ and that of type II error is $\left(1-q_{1}\right)\left(1-q_{2}\right)$. The combination of these two probabilities indicates the quality of auditing. The quality of auditing can be improved if the external auditor expends effort. However, it is not costless. The cost of auditing increases as the external auditor increases $q_{1}$ and as he decreases $q_{2}$. We denote the cost function of auditing as $C\left(q_{1}, q_{2}\right)$ where $C \geq 0, \partial C / \partial q_{1} \geq 0, \partial C / \partial q_{2} \leq 0$. We assume that the principal always cover the cost of auditing. Therefore, the individual rationality condition of the external auditor is always satisfied.

When the external auditor interprets or misinterprets that the agent has manipulated, the agent must bear the penalty. We denote the amount of penalty as $0 \leq f \leq \bar{f}$ where $\bar{f}$ is the upper limit of penalty that is pre-decided by the rules outside our model. The principal receives penalty earnings. The penalty may be pecuniary one (e.g., budget cut), but may be non-pecuniary one (e.g., administrative disposition). When the penalty is pecuniary one, all the penalty earnings are added to the benefit of the principal. If the penalty is non-pecuniary one, only part of the penalty earnings is added to the benefit of the principal. We denote the portion of the penalty to be added as $0 \leq \kappa \leq 1$.

\subsection{Timing}

The timing of our principal=agent=external auditor model consists of nine steps (see Figure 1). Firstly, the principal offers a contract to the agent. This contract prescribes the amount of penalty imposed on the agent if the auditor concludes that the agent has manipulated. Secondly, the agent accepts (or rejects) the contract offered by the principal if he judges that the expected private interest that will happen is above (below) the expected penalty burden. Thirdly, the nature selects one of the four cases. Fourthly, the agent performs project evaluation using the two models. Fifth, the agent selects one of the two results of the analysis of project evaluation and reports it to the principal. Sixth, one of the two alternatives is implemented based on the report of the project evaluation. Seventh, the external auditor makes the inference on the analysis of project evaluation. Eighth, the external auditor reports the result of the audit. Ninth, if the external auditor judges that the agent has manipulated, the penalties are imposed on the agent and are transferred from the agent to the principal. The cost of auditing is also transferred from the principal to the external auditor.

The game tree of our model is described in Figure 2. The agent can select the result of project evaluation reported to the principal with knowledge on the case that realized. On the other hand, the principal and the external auditor have no information about the case that realized and the model that the agent selected when the external auditor perform auditing. This asymmetry of information is key point of our model analysis.

\subsection{Optimal external audit problem}

The objective of the external audit system is to maximize the total benefit of the principal. It consists of three parts,

\begin{tabular}{|c|c|c|c|c|c|}
\hline $\begin{array}{l}\text { P offers } \\
\text { a contract } \\
\text { to } \mathrm{A}\end{array}$ & $\begin{array}{l}\text { A accepts } \\
\text { (or rejects) } \\
\text { the contract }\end{array}$ & $\begin{array}{l}\mathrm{N} \text { selects } \\
\text { one of } \\
\text { the four cases }\end{array}$ & $\begin{array}{c}\text { A does } \\
\text { project } \\
\text { evaluation }\end{array}$ & \multirow{5}{*}{5} & \multirow{5}{*}{$\begin{array}{l}\text { A selects } \\
\text { one of } \\
\text { the results of } \\
\text { project } \\
\text { evaluation } \\
\text { and reports it }\end{array}$} \\
\hline 1 & 2 & 3 & 4 & & \\
\hline & 1 & 1 & +1 & & \\
\hline 9 & 8 & 7 & 6 & & \\
\hline $\begin{array}{l}\text { Penalty is } \\
\text { transferred } \\
\text { from A to P. } \\
\text { Cost of auditing } \\
\text { is transferred } \\
\text { from P to EA }\end{array}$ & $\begin{array}{l}\text { EA reports } \\
\text { the result } \\
\text { of auditing }\end{array}$ & $\begin{array}{l}\text { EA checks } \\
\text { the analysis } \\
\text { of project } \\
\text { evaluation }\end{array}$ & $\begin{array}{l}\text { One of } \\
\text { the alternatives } \\
\text { is implemented }\end{array}$ & & \\
\hline
\end{tabular}

Fig. 1. Timing of the model. 


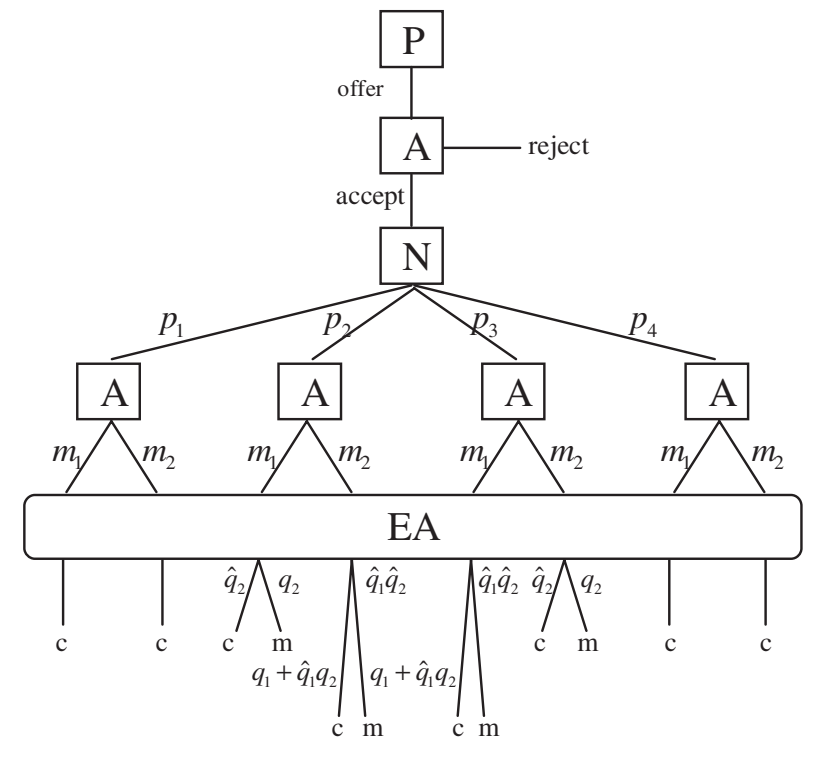

*where $\hat{q}_{1} \equiv 1-\hat{q}_{1}, \hat{q}_{2} \equiv 1-\hat{q}_{2}$, c:correct interpretation, m:misinterpretation

Fig. 2. Game tree*.

namely, the NB of the public investment project, the penalty earnings transferred from the agent and the cost of auditing transferred to the external auditor. The NB depends on which of the four cases is realized and whether the agent manipulates or not. The penalty earnings depends on which of the four cases is realized, whether the agent manipulates or not, and whether the external auditor judges that the agent has manipulated or not. The cost of auditing depends on the audit quality that the principal prescribes. Now, to simplify the expression, we define the following five variables: $p_{23} \equiv p_{2}+p_{3}, E^{H}[B] \equiv\left(1-p_{4}\right) B_{1}+p_{4} B_{2}, E^{L}[B] \equiv p_{1} B_{1}+\left(1-p_{1}\right) B_{2}, E^{H}[R] \equiv p_{1} R_{1}+\left(1-p_{1}\right) R_{2}$ and $E^{L}[R] \equiv\left(1-p_{4}\right) R_{1}+p_{4} R_{2}$. Consequently, the expected benefit of the principal can be written as

$$
u_{P}^{M}\left(q_{1}, q_{2}, f\right) \equiv E^{L}[B]+p_{23}\left\{q_{1}+\left(1-q_{1}\right) q_{2}\right\} \kappa f-C\left(q_{1}, q_{2}\right)
$$

when the agent manipulates, and as

$$
u_{P}^{N M}\left(q_{1}, q_{2}, f\right) \equiv E^{H}[B]+p_{23} q_{2} \kappa f-C\left(q_{1}, q_{2}\right)
$$

when the agent does not manipulate.

The payoff of the agent consists of two parts, that is, the private interest from the public investment project and the penalty burden. The former depends on which of the four cases is realized and whether the agent manipulates or not. The latter depends on which of the four cases is realized, whether the agent manipulates or not, and whether the auditor judges that the agent has manipulated or not. The payoff of the agent can be written as

$$
u_{A}^{M}\left(q_{1}, q_{2}, f\right) \equiv E^{H}[R]-p_{23}\left\{q_{1}+\left(1-q_{1}\right) q_{2}\right\} f
$$

when he manipulates, and as

$$
u_{A}^{N M}\left(q_{1}, q_{2}, f\right) \equiv E^{L}[R]-p_{23} q_{2} f
$$

when he does not manipulate. Whether the agent manipulates or not depends on the magnitude relationship between $u_{A}^{M}\left(q_{1}, q_{2}, f\right)$ and $u_{A}^{N M}\left(q_{1}, q_{2}, f\right)$. If the former is larger than the latter and is larger than zero (which is the reservation payoff of the agent), the agent accepts the contract and manipulates in case 2 and case 3 . On the other hand, If the latter is larger than the former and is larger than zero, the agent accepts the contract and does not manipulate in all the four cases.

We are now at the stage of defining the optimal external audit system. Before defining it, we had better specify the cost function of auditing as

$$
C\left(q_{1}, q_{2}\right)=\theta_{1} q_{1}+\theta_{2}\left(\bar{q}_{2}-q_{2}\right)
$$

where $\theta_{1}, \theta_{2} \geq 0$. This simple cost function is assumed to do model analysis analytically. The principal can control three variables: the penalty $f$ and the probabilities $q_{1}$ and $q_{2}$. The optimal structure of the external audit system can be defined as the solution of the following two-step optimization problem. We call this problem as the optimal external audit problem. 
Optimal External Audit Problem

$$
\max \left\{u_{P}^{M}\left(q_{1}^{M}, q_{2}^{M}, f^{M}\right), u_{p}^{N M}\left(q_{1}^{N M}, q_{2}^{N M}, f^{N M}\right)\right\}
$$

where

$$
\begin{aligned}
& u_{A}^{M}\left(q_{1}^{M}, q_{2}^{M}, f^{M}\right) \equiv \operatorname{Max}_{q_{1}, q_{2}, f} E^{L}[B]+p_{23}\left\{q_{1}+\left(1-q_{1}\right) q_{2}\right\} \kappa f-\theta_{1} q_{1}-\theta_{2}\left(\bar{q}_{2}-q_{2}\right) \\
& \text { s.t. }(\mathrm{IR}-\mathrm{M}) E^{H}[R]-p_{23}\left\{q_{1}+\left(1-q_{1}\right) q_{2}\right\} f \geq 0, \\
& \quad(\mathrm{IC}-\mathrm{M}) \Delta R-q_{1}\left(1-q_{2}\right) f \geq 0, \\
& \quad \text { (P) } 0 \leq f \leq \bar{f}, \quad(\mathrm{Q} 1) 0 \leq q_{1} \leq 1, \quad(\mathrm{Q} 2) 0 \leq q_{2} \leq \bar{q}_{2} \\
& u_{P}^{N M}\left(q_{1}^{N M}, q_{2}^{N M}, f^{N M}\right) \equiv \operatorname{Max}_{q_{1}, q_{2}, f} E^{H}[B]+p_{23} q_{2} \kappa f-\theta_{1} q_{1}-\theta_{2}\left(\bar{q}_{2}-q_{2}\right) \\
& \text { s.t. }(\mathrm{IR}-\mathrm{NM}) E^{L}[R]-p_{23} q_{2} f \geq 0, \\
& \text { (IC-NM) } \Delta R-q_{1}\left(1-q_{2}\right) f<0, \\
& \text { (P) } 0 \leq f \leq \bar{f}, \quad \text { (Q1) } 0 \leq q_{1} \leq 1, \quad \text { (Q2) } 0 \leq q_{2} \leq \bar{q}_{2}
\end{aligned}
$$

The optimal external audit problem consists of three sub optimization problems. First is the optimization problem to define the optimal structure of the external audit system under the condition that the agent manipulates [eq. (7)]. Next is the optimization problem to define the optimal structure of the external audit system under the condition that the agent does not manipulate [eq. (8)]. Finally, the optimization problem is to compare the optimal structure with manipulation and that of without manipulation and to select the optimal scheme [eq. (6)].

\section{Model Analysis}

\subsection{Solution strategy}

The external audit problem defined in the previous section has to be solved in order to derive the optimal structure of the external audit system. We solve it following a three-step procedure. Firstly, we solve the optimization problems with the constraint of manipulation. Secondly, we solve that with the constraint of no manipulation. Thirdly, we compare two solutions and select the optimal scheme.

Before solving the problem, we must confirm that only one of the following two conditions is always satisfied (see Appendix).

$$
\begin{aligned}
& \frac{E^{L}[R]}{p_{23} \bar{q}_{2}}<\frac{E^{H}[R]}{p_{23}}<\frac{\Delta R}{1-\bar{q}_{2}} \\
& \frac{\Delta R}{1-\bar{q}_{2}} \leq \frac{E^{H}[R]}{p_{23}} \leq \frac{E^{L}[R]}{p_{23} \bar{q}_{2}}
\end{aligned}
$$

The three terms in these equations are related to the private interests of the agent. As we can see from IC-M and IC-NM of the optimal external audit problem, it is relatively easy to prevent the manipulation when the former inequality is satisfied. The solution of the external audit problem differs depending on which of the two conditions is satisfied. Hereafter, we call eq. (11) as the condition $\alpha$ and eq. (12) as the condition $\beta$.

\subsection{Optimal structure with manipulation}

The solution of the optimization problem under the constraint that the agent manipulates is summarized in Table 2 and Figure 3 (as for the formal analysis, see Appendix). Figure 4 shows the optimal structure of the external audit system with manipulation in some special cases: $\bar{q}_{2}=0, \bar{q}_{1}=1, \theta_{2}=0$ and $\theta_{2} \rightarrow \infty$. The optimal audit quality and penalty differ depending on the parameters of the problem. In each region in Figure 3, they differ qualitatively.

As shown in Table 2, the probability $q_{2}$ is equal to $\bar{q}_{2}$ in all the regions. The results show that the probability of type I error is maximized to get the penalty earnings as much as possible and to cut the auditing cost. Consequently, the size of the parameter $\theta_{2}$, which means the cost efficiency of decreasing the probability of type I error, does not have any impact on the optimal structure of the external audit system with manipulation. On the other hand, the probability $q_{1}$ is determined as a result of comparison between the increment of the penalty earnings and that of the auditing cost, since there is a tradeoff relationship between the two. In region 2 and region 4, the external auditor does not expend any efforts for auditing. However, it should be noted that this result does not mean that there is no need for the external auditor, since the principal cannot get penalty earning if the external auditor does not exist. When $\bar{q}_{2}=1$, the effort of the external auditor become zero for any $\left(\theta_{1}, \bar{f}\right)$. In region 3 and region 4, IR-M is binding. Especially in region 4 , the penalty is below the upper limit $\bar{f}$ because of IR-M. As shown in Figure 4(a), if the probability of type I error is zero $a$ priori $\left(\bar{q}_{2}=0\right)$, the IR-M is not binding for any $\left(\theta_{1}, \bar{f}\right)$. 
[condition $\alpha$ ]

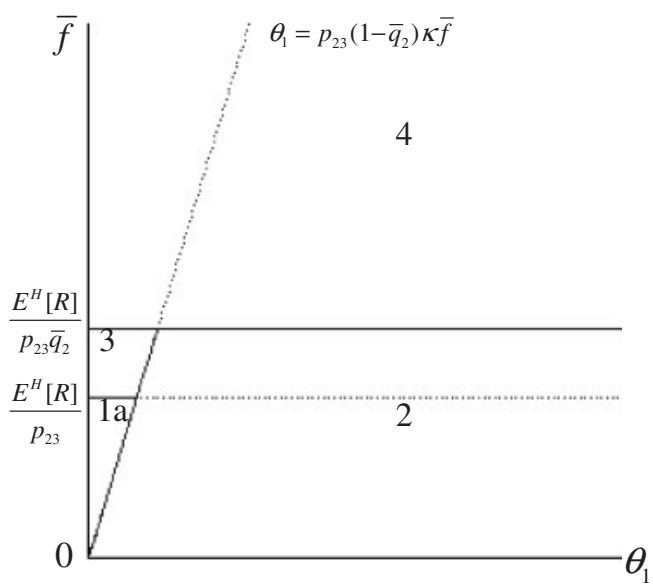

[condition $\beta$ ]

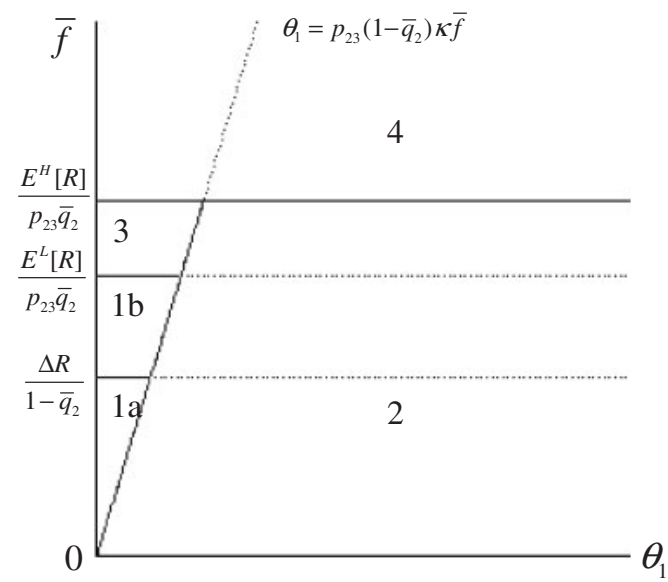

Fig. 3. Optimal structure with manipulation: diagram.

[condition $\alpha$ ]

a) $\bar{q}_{2}=0$

b) $\bar{q}_{2}=1$

c) $\bar{\theta}_{2}=0$, d) $\bar{\theta}_{2}=\infty$

$$
\begin{gathered}
\text { condition } \alpha \\
\text { is not satisfied }
\end{gathered}
$$

4

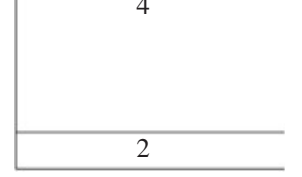

4

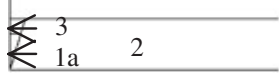

[condition $\beta$ ]

$1 b$

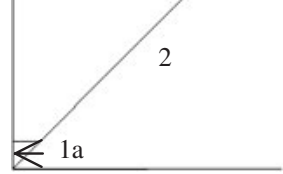

condition $\beta$

is not satisfied

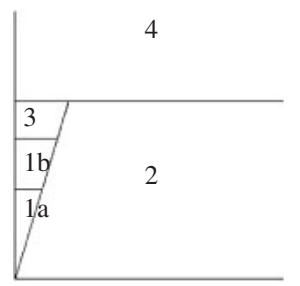

Fig. 4. Optimal structure with manipulation: special cases.

Table 2. Optimal structure with manipulation: mathematical formula.

\begin{tabular}{lcccccc}
\hline & $q_{1}^{M}$ & $q_{2}^{M}$ & $f^{M}$ & IR-M* & IC-M* & $u_{P}^{M}\left(q_{1}^{M}, q_{2}^{M}, f^{M}\right)$ \\
\hline region 1a & 1 & $\bar{q}_{2}$ & $\bar{f}$ & $\mathrm{~N}$ & $\mathrm{~N}$ & $E^{L}[B]+p_{23} \kappa \bar{f}-\theta_{1}$ \\
region 1b & $\Delta R$ & $\bar{q}_{2}$ & $\bar{f}$ & $\mathrm{~N}$ & $\mathrm{~B}$ & $E^{L}[B]+p_{23} \bar{q}_{2} \kappa \bar{f}+p_{23} \kappa \Delta R-\theta_{1} \frac{\Delta R}{\left(1-\bar{q}_{2}\right) \bar{f}}$ \\
region 2 & 0 & $\bar{q}_{2}$ & $\bar{f}$ & $\mathrm{~N}$ & $\mathrm{~N}$ & $E^{L}[B]+p_{23} \bar{q}_{2} \kappa \bar{f}$ \\
region 3 & $\frac{E^{H}[R]-p_{23} \bar{q}_{2} \bar{f}}{p_{23}\left(1-\bar{q}_{2}\right) \bar{f}}$ & $\bar{q}_{2}$ & $\bar{f}$ & $\mathrm{~B}$ & $\mathrm{~N}$ & $E^{L}[B]+\kappa E^{H}[R]-\theta_{1} \frac{E^{H}[R]-p_{23} \bar{q}_{2} \bar{f}}{p_{23}\left(1-\bar{q}_{2}\right) \bar{f}}$ \\
region 4 & 0 & $\bar{q}_{2}$ & $\frac{E^{H}[R]}{p_{23} \bar{q}_{2}}$ & $\mathrm{~B}$ & $\mathrm{~N}$ & $E^{L}[B]+\kappa E^{H}[R]$ \\
\hline
\end{tabular}

*B: binding, N: non-binding

\subsection{Optimal structure without manipulation}

The solution of optimization problem under the constraint that the agent does not manipulate is summarized in Table 3 and Figure 5 (as for the formal analysis, see Appendix). Figure 6 shows the optimal structure of the external 
Table 3. Optimal structure without manipulation: mathematical formula.

\begin{tabular}{lcccccc}
\hline & $q_{1}^{N M}$ & $q_{2}^{N M}$ & $f^{N M}$ & IR-NM* & IC-NM* & $u_{P}^{N M}\left(q_{1}^{N M}, q_{2}^{N M}, f^{N M}\right)$ \\
\hline region 0 & $n . s$. & $n . s$. & $n . s$. & - & - & $n . s$. \\
region 1a & 1 & $\frac{\bar{f}-\Delta R}{\bar{f}}$ & $\bar{f}$ & $\mathrm{~N}$ & $\mathrm{~B}$ & $E^{H}[B]+\left(p_{23} \kappa \bar{f}+\theta_{2}\right) \frac{\bar{f}-\Delta R}{\bar{f}}-\theta_{1}-\theta_{2} \bar{q}_{2}$ \\
region 1b & $\frac{\Delta R}{\left(1-\bar{q}_{2}\right) \bar{f}}$ & $\bar{q}_{2}$ & $\bar{f}$ & $\mathrm{~N}$ & $\mathrm{~B}$ & $E^{H}[B]+\left(p_{23} \kappa \bar{f}+\theta_{2}\right) \bar{q}_{2}-\theta \frac{\Delta R}{\left(1-\bar{q}_{2}\right) \bar{f}}-\theta_{2} \bar{q}_{2}$ \\
region 2 & $\frac{\Delta R}{\left(1-q_{2}^{N M}\right) \bar{f}}$ & $1-\sqrt{\frac{\theta_{1}}{p_{23} k \bar{f}+\theta_{2}}} \frac{\Delta R}{\bar{f}}$ & $\bar{f}$ & $\mathrm{~N}$ & $\mathrm{~B}$ & $E^{H}[B]+\left(p_{23} \kappa \bar{f}+\theta_{2}\right) q_{2}^{N M}-\theta_{1} \frac{\Delta R}{\left(1-q_{2}^{N M}\right) \bar{f}}-\theta_{2} \bar{q}_{2}$ \\
region 3 & $\frac{\Delta R}{\bar{f}}$ & 0 & $\bar{f}$ & $\mathrm{~N}$ & $\mathrm{~B}$ & $E^{H}[B]+\left(p_{23} \kappa \bar{f}+\theta_{2}\right) \times 0-\theta_{1} \frac{\Delta R}{\bar{f}}-\theta_{2} \bar{q}_{2}$ \\
region 4 & $\min \left\{1, \frac{\bar{q}_{2}}{1-\bar{q}_{2}} \frac{p_{23} \Delta R}{E^{L}[R]}\right\}$ & $\min \left\{\frac{E^{L}[R]}{E^{H}[R]}, \bar{q}_{2}\right\}$ & $\max \left\{\frac{E^{H}[R]}{p_{23}}, \frac{E^{L}[R]}{p_{23} \bar{q}_{2}}\right\}$ & $\mathrm{B}$ & $\mathrm{B}$ & $E^{H}[B]+\kappa E^{L}[R]-\theta_{1} q_{1}^{N M}-\theta_{2}\left(\bar{q}_{2}-q_{2}^{N M}\right)$ \\
region 5 & $\frac{q_{2}^{N M}}{1-q_{2}^{N M}} \frac{p_{23} \Delta R}{E^{L}[R]}$ & $1-\sqrt{\frac{\theta_{1}}{\theta_{2}}} \frac{p_{23} \Delta R}{E^{L}[R]}$ & $\frac{E^{L}[R]}{p_{23} q_{2}^{N M}}$ & $\mathrm{~B}$ & $\mathrm{~B}$ & $E^{H}[B]+\kappa E^{L}[R]-\theta_{1} \frac{q_{2}^{N M}}{1-q_{2}^{N M}} \frac{p_{23} \Delta R}{E^{L}[R]}-\theta_{2}\left(\bar{q}_{2}-q_{2}^{N M}\right)$ \\
region 6 & $\frac{p_{23} \Delta R}{p_{23} \bar{f}-E^{L}[R]}$ & $\frac{E^{L}[R]}{p_{23} \bar{f}}$ & $\bar{f}^{L}$ & $\mathrm{~B}$ & $\mathrm{~B}$ & $E^{H}[B]+\kappa E^{L}[R]-\theta_{1} \frac{p_{23} \Delta R}{p_{23} \bar{f}-E^{L}[R]}-\theta_{2}\left(\bar{q}_{2}-\frac{E^{L}[R]}{p_{23} \bar{f}}\right)$ \\
\hline
\end{tabular}

*B: binding, $\mathrm{N}$ : non-binding, n.s.: no solution

[condition $\alpha$ ]

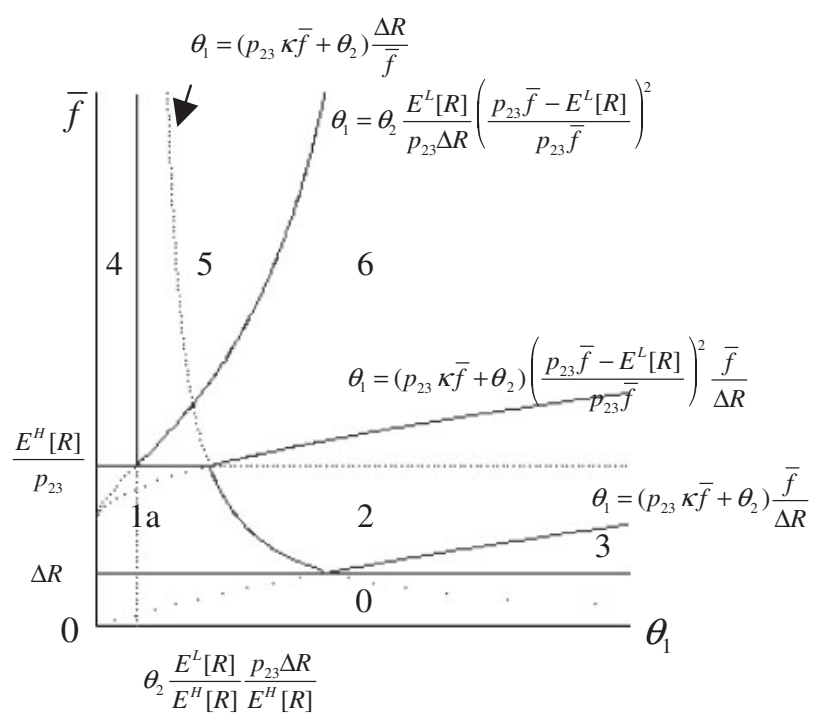

[condition $\beta$ ]

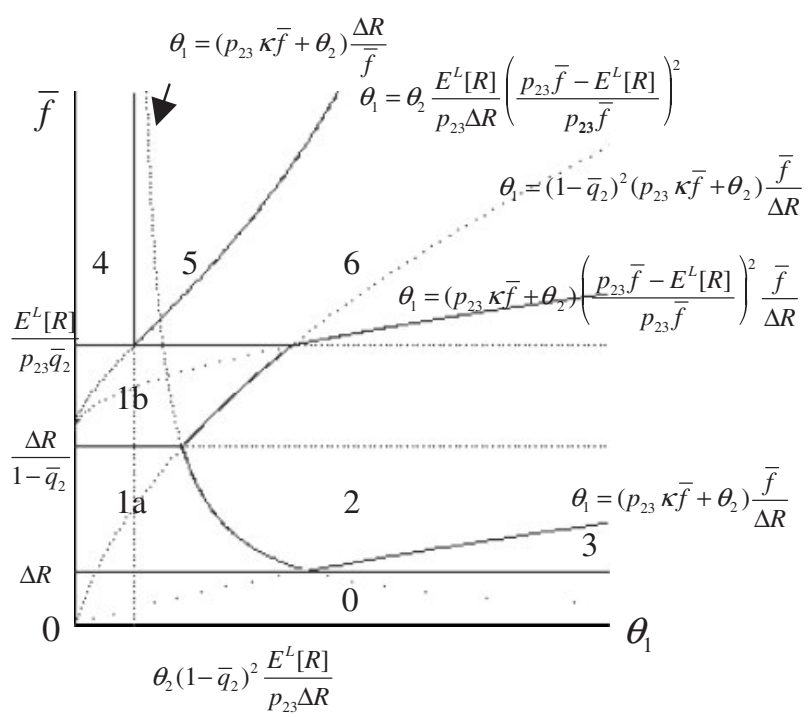

Fig. 5. Optimal structure without manipulation: diagram.

audit system without manipulation in some special cases: $\bar{q}_{2}=0, \bar{q}_{2}=1, \theta_{2}=0$ and $\theta_{2} \rightarrow \infty$. As is the case with manipulation, the optimal audit quality and penalty differ depending on the parameters of the problem and differ qualitatively in each region in Figure 5.

As shown in Table 3, IC-NM is binding for all the regions except region 0 where there is no solution since the upper limit of penalty is too low to prevent the manipulation. When the agent does not manipulate, the penalty earnings does not change even if the principal increases in the contract. Therefore, the probability is minimized to cut the auditing cost on condition that IC-NM is satisfied. This is the reason why IC-NM is binding. As IC-NM, $q_{1}$ is strictly positive in all the regions. Therefore, in contrary to the case where the agent manipulates, he must expend some effort to prevent the manipulation. The probability of type I error $q_{2}$ is not always equal to $\bar{q}_{2}$. If $q_{2}$ increases, $q_{1}$ also increases since ICNM is binding. Furthermore, IR-NM is also binding in region 4, region 5 and region 6 . The probability of type I error is determined as a result of comparison between the penalty earnings and the cost of auditing within the limits of the constrained conditions. As shown in Table 3 and Figure 6(a), if the probability of type I error is zero a priori $\left(\bar{q}_{2}=0\right)$, the IR-NM is not binding for any $\left(\theta_{1}, \bar{f}\right)$ and the audit quality $\left(q_{1}, q_{2}\right)$ is same for any $\theta_{1}$.

The size of the parameter $\theta_{2}$ affects the optimal structure of the external audit system without manipulation. When $\theta_{2}$ goes to infinity, in all regions where $q_{2}$ is not $\bar{q}_{2}$, the expected benefit of the principal goes to minus infinity (see Table 3). On the other hand, the expected benefit of the principal under the constraint that the agent manipulates is always positive (see Table 2). Therefore, we can see from Figure 6(d) that the principal should not prevent the manipulation for any $\left(\theta_{1}, \bar{f}\right)$ when $\theta_{2}$ goes to infinity and the condition $\alpha$ is satisfied. In these cases, the increase of the upper limit of the penalty is not enough to prevent the manipulation. The improvement of the audit technology or that of the accuracy of the project evaluation are needed so that $\bar{q}_{2}, \theta_{2}$ or $p_{23}$ decreases.

From Figure 4(a) and Figure 6(a), we can see that the agent's individual rationality condition is not binding for any $\left(\theta_{1}, \bar{f}\right)$ when type I error of the external auditor is zero $\left(\bar{q}_{2}=0\right)$. It is interesting that the individual rationality condition becomes important only when the probability of type I error is strictly positive. As we have mentioned in the second 
[condition $\alpha$ ]

a) $\bar{q}_{2}=0$

b) $\bar{q}_{2}=1$

c) $\bar{\theta}_{2}=0$

d) $\bar{\theta}_{2}=\infty$

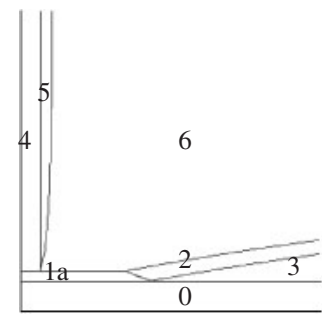

6

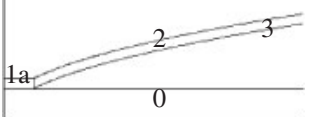

condition $\beta$

is not satisfied
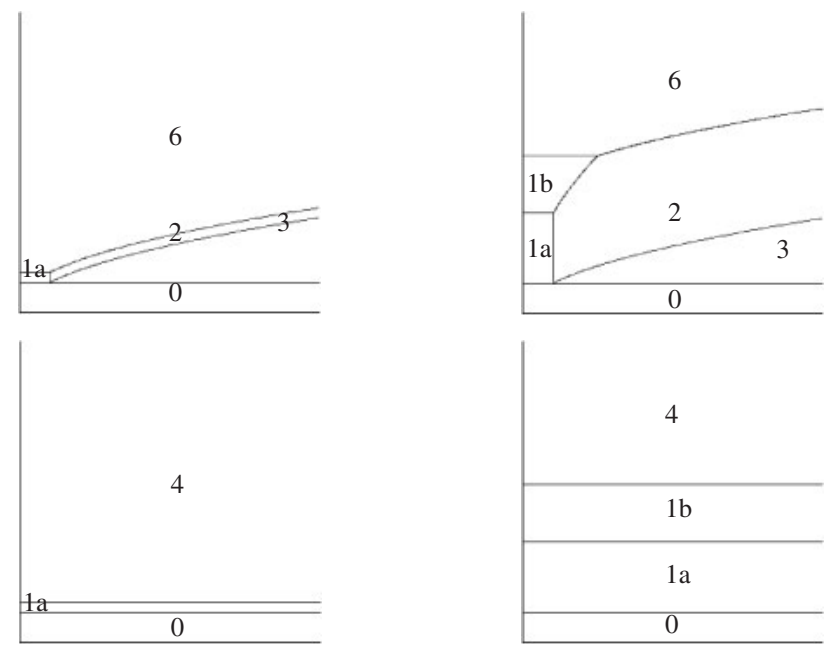

Fig. 6. Optimal structure without manipulation: special cases.

section, our precedent paper (Fukumoto and Tsuchiya, 2004) ignored the agent's individual rationality condition and the possibility of type I error. Since its analysis was limited to the case of $\bar{q}_{2}=0$, we can say that its result is still consistent within the scope of analysis.

\subsection{Optimal scheme}

By comparing two solutions, we can derive the optimal scheme of the optimal external audit problem. It is summarized in Figure 7 (as for the formal analysis, see Appendix). There are three qualitative different patterns of the

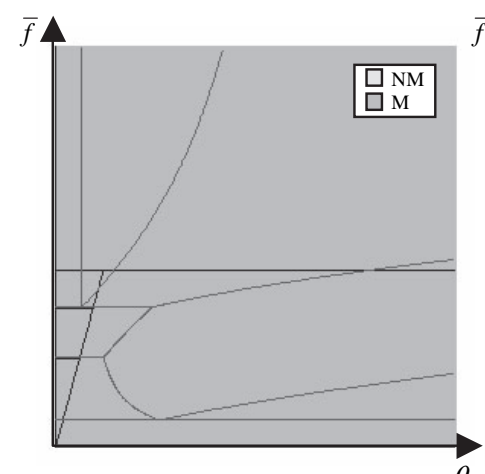

(a) $\Psi<\max -\bar{q}_{2} \theta_{2},-\frac{E^{L}[R]}{E^{H}[R]} \theta_{2}$

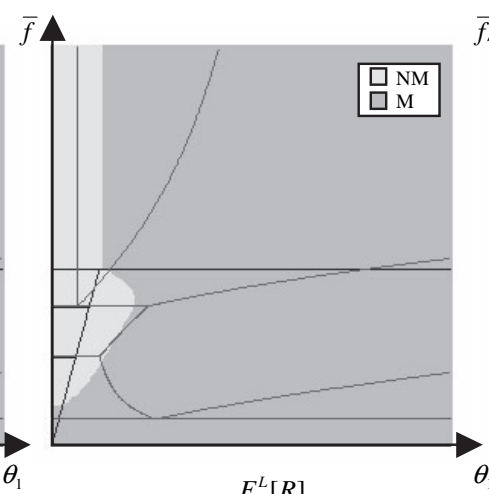

(b) $\max -\bar{q}_{2} \theta_{2},-\frac{E^{L}[R]}{E^{H}[R]} \theta_{2} \leq \Psi<0$

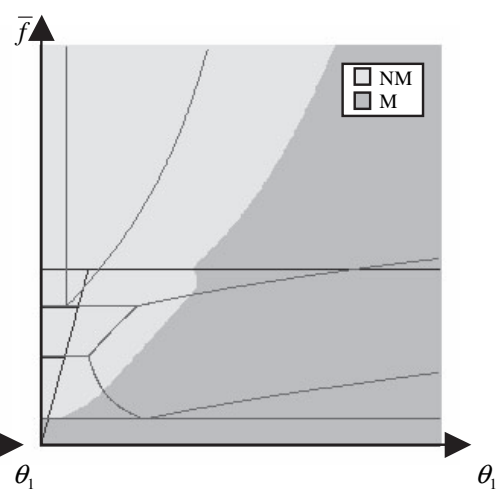

(c) $0 \leq \Psi$

Fig. 7. Optimal scheme in the space of $\left(\theta_{1}, \bar{f}\right)$. 
optimal scheme in the field of $\left(\theta_{1}, \bar{f}\right)$, which depends on the size of $\Psi \equiv p_{23}(\Delta B-\kappa \Delta R)-\theta_{2} \bar{q}_{2}$. The first term of $\Psi$ comes from the fact that the NB of the principal increases and the penalty earnings decreases when the principal prevents the manipulation. Second term of $\Psi$ comes from the fact that when the principal prevents the manipulation the cost of auditing increases to decrease the probability of type I error.

In the first pattern $\left(\Psi<\max \left\{-\bar{q}_{2} \theta_{2},-\left(E^{L}[R] / E^{H}[R] \theta_{2}\right\}\right)\right.$, the principal should make the agent manipulate and increase the penalty earnings as much as possible for any $\left(\theta_{1}, \bar{f}\right)$. In the second pattern $\left(\max \left\{-\bar{q}_{2} \theta_{2}\right.\right.$, $\left.\left.-\left(E^{L}[R] / E^{H}[R]\right) \theta_{2}\right\} \leq \Psi<0\right)$, the principal should make the agent manipulate if $\theta_{1}$ is sufficiently small and $\bar{f}$ is sufficiently large, and prevent the manipulation otherwise. In this pattern, if $\theta_{1}$ is not sufficiently small, the principal should not prevent the manipulation no matter what the size of the upper limit of the penalty is. This pattern does not appear if the probability of type I error or the cost of decreasing it is zero. In the third pattern $(0 \leq \Psi)$, the principal should prevent the manipulation if $\bar{f}$ is sufficiently large and $\theta_{1}$ is sufficiently small. In this pattern, there is some positive value for any $\theta_{1}$ such that, if the upper limit of the penalty is above which, the principal should prevent the manipulation.

From the results above, we can say that it is important for the principal to know the parameter configuration and to prepare the optimal contract. However, we have to be heedful as the decrease of the social reliability on the project evaluation system that happens when the agent manipulates is not considered in the model. If the social reliability decreases, the implementation of socially desirable projects would be hindered. Such kind of social loss has a huge impact. Therefore, we must expend more efforts in preventing the agent's manipulation.

The parameters in the model change as the technology of auditing or of project evaluation improves. If the procedure of analysis is standardized or if the disclosure of the information on the analysis is improved, it becomes easier for the external auditor to increase the quality of audit. These changes can be expressed as the decrease of $\theta_{1}, \theta_{2}$ or $\bar{q}_{2}$. In these cases, not only the benefit of the principal increases, but also the optimal scheme for certain parameter configurations changes from the scheme with manipulation to that without manipulation. We can infer that there is a complementary relationship between the design of the optimal contract and the development of the technology of auditing.

\section{Concluding Remarks}

We have analyzed the optimal external audit system focusing on the possibility of misinterpretation by the external auditor. We have shown that we must consider the agent's individual rationality condition when there is the possibility of misinterpretation and that the effectiveness of increasing the upper limit of the penalty decreases when the individual rationality condition is binding. Furthermore, we have also investigated the complementary relationship between the design of the optimal contract and the development of the technology of auditing or of project evaluation.

Our model can be extended to a variety of directions. Though we have considered the official auditing institutions such as AEB in mind, we can take the unofficial auditing function of the mass media into consideration. It is likely that the mass media points out the agent's manipulation without possessing sufficient knowledge with regards to the matter. The impact of their unofficial auditing function is so large that it is worth analyzing. They have strong driving factors (for example, public expectations) to point out the agent's manipulation, but they do not have enough incentive and ability to decrease the probability of misinterpretation. Since the principal can not close a contract with them directly, we can not use the optimal external audit system described above. We must find another incentive mechanism that controls both the good and bad impacts of their auditing function. We can also take the auditing function of some academic researchers into consideration. They have enough knowledge on the analysis of project evaluation and ability to improve the quality of auditing. Though, in reality, they do not participate fully in the audit of the analysis of project evaluation. One reason is that even if they perform accurate auditing, they can not get high academic appreciation from the academic society. As in the case of the mass media, we must introduce another incentive mechanism to use their knowledge and ability effectively.

Another direction is to seek for the optimal audit system in the context of a dynamic working framework. The social reliability on the project evaluation changes as time goes by. The technology of auditing and of project evaluation also improves with time. Furthermore, in the dynamic context, it becomes possible to use some incentive mechanism such as the reputation mechanism, which cannot be used in the static framework. Therefore, there is a possibility that the structure of the optimal audit system will change drastically from that described above. Though its analysis is complicated, we have confidence that the necessity of it is high.

\section{Appendix}

\section{Derivation of Eq. (11) and Eq. (12)}

As for the two terms $\Delta R /\left(1-\bar{q}_{2}\right)$ and $E^{H}[R] / p_{23}$, only one of the following two conditions is always satisfied: $\Delta R /\left(1-\bar{q}_{2}\right) \leq E^{H}[R] / p_{23}$ or $E^{H}[R] / p_{23}<\Delta R /\left(1-\bar{q}_{2}\right)$. When $\Delta R /\left(1-\bar{q}_{2}\right) \leq E^{H}[R] / p_{23}$ is satisfied, we can derive $E^{H}[R] / p_{23} \leq \bar{E}^{H}[R] / p_{23} / \bar{q}_{2}$ since $E^{H}[R]-E^{L}[R]=p_{23} \Delta R$. As the same way, we can derive $E^{H}[R] / p_{23} / \bar{q}_{2}<$ $E^{H}[R] / p_{23}$ from $E^{H}[R] / p_{23}<\Delta R /\left(1-\bar{q}_{2}\right)$. Therefore, it is confirmed that only one of eq. (11) and eq. (12) is always satisfied. 


\section{Process to Derive Table 2 and Figure 3}

We distinguish the case where IR-M is not binding and that where IR-M is binding.

In the case where IR-M is not binding, it is clear that $q_{2}^{M}<\bar{q}_{2}$ contradicts with the definition of the optimal solution. Therefore, $q_{2}^{M}=\bar{q}_{2}$. If we increase $f$ keeping $q_{1} f$ constant, the objective function increases though the other constrained conditions do not change. Therefore, $f^{M}=\bar{f}$. The first order derivative of the objective function is $p_{23}(1-$ $\left.\bar{q}_{2}\right) \kappa \bar{f}-\theta_{1}$. Therefore, $q_{1}^{M}$ is the minimal value which satisfies all the other constrained conditions when $p_{23}(1-$ $\left.\bar{q}_{2}\right) \kappa \bar{f}-\theta_{1}<0$ and is the maximal value which satisfies all the other constrained conditions when $p_{23}\left(1-\bar{q}_{2}\right) \kappa \bar{f}-$ $\theta_{1}>0$. From IC-NM, $q_{1} \leq \frac{\Delta R}{\left(1-\bar{q}_{2}\right) \bar{f}}$. From IR-M, $q_{1}<\frac{E^{H}[R]-p_{23} \bar{q}_{2} \bar{f}}{p_{23}\left(1-\bar{q}_{2}\right) \bar{f}}$. From Q1, $0 \leq q_{1} \leq 1$. Therefore, $q_{1}^{M}$ depends on the sign of $p_{23}\left(1-\bar{q}_{2}\right) \kappa \bar{f}-\theta_{1}$ and the magnitude relationship among $0,1, \frac{\Delta R}{\left(1-\bar{q}_{2}\right) \bar{f}}$ and $\frac{E^{H}[R]-p_{23} \bar{q}_{2} \bar{f}}{p_{23}\left(1-\bar{q}_{2}\right) \bar{f}}$. There are two patterns as for the sign of $p_{23}\left(1-\bar{q}_{2}\right) \kappa \bar{f}-\theta_{1}$ and are twelve patterns as for the permutaion of the four terms. By examining all the twenty four (two times twelve) patterns, we can derive $q_{1}^{M}$.

In the case where IR-M is binding, $f=\frac{E^{H}[R]}{p_{23}\left\{q_{1}+\left(1-q_{1}\right) q_{2}\right\}}$. It is clear that $q_{2}^{M}<\bar{q}_{2}$ contradicts with the definition of the optimal solution. Therefore, $q_{2}^{M}=\bar{q}_{2}$. It is also clear that $q_{1}^{M}$ is the minimal value which satisfies all the other constrained conditions. From $\mathrm{P}, q_{1} \geq \frac{E^{H}[R]-p_{23} \bar{q}_{2} \bar{f}}{p_{23}\left(1-\bar{q}_{2}\right) \bar{f}}$. From IC-M, $q_{1} \leq \frac{p_{23} \bar{q}_{2} \Delta R}{\left(1-\bar{q}_{2}\right) E^{L}[R]}$. From $\mathrm{Q} 1,0 \leq q_{1} \leq 1$. Therefore, $q_{1}^{M}$ depends on the magnitude relationship among $0,1, \frac{E^{H}[R]-p_{23} \bar{q}_{2} \bar{f}}{p_{23}\left(1-\bar{q}_{2}\right) \bar{f}}$ and $\frac{p_{23} \bar{q}_{2} \Delta R}{\left(1-\bar{q}_{2}\right) E^{L}[R]}$. By examining all the twelve patterns, we can derive $q_{1}^{M}$.

By comparing the optimal solution when IR-M is binding and that when IR-M is not binding, we can derive the true optimal solution as shown in Table 2 and Figure 3.

\section{Process to Derive Table 3 and Figure 5}

We distinguish the case where IR-NM is not binding and that where IR-NM is binding.

In the case where IR-NM is not binding, it is clear that $f^{N M}<\bar{f}$ contradicts with the definition of the optimal solution. Therefore, $f^{N M}=\bar{f}$. It is also clear that $q_{1}=\frac{\Delta R}{\left(1-q_{2}\right) \bar{f}}$ satisfies IC-NM and maximizes the objective function. From Q2, $0 \leq q_{2} \leq 1$. The first order derivative of the objective function is $\Xi\left(q_{2}\right) \equiv\left(p_{23} \kappa \bar{f}+\theta_{2}\right)-\theta_{1} \frac{\Delta R}{\bar{f}} \frac{1}{\left(1-q_{2}\right)^{2}}$ where $\frac{\partial \Xi}{\partial q^{2}}<0$. Therefore, the optimal solution of $q_{2}$ depends on which of the three conditions, that is, $0>\Xi(0)>\Xi\left(\bar{q}_{2}\right)$, $\Xi(0)>0>\Xi\left(\bar{q}_{2}\right)$ or $\Xi(0)>\Xi\left(\bar{q}_{2}\right)>0$ is satisfied. From $\Xi\left(q_{2}\right)=0, q_{2}=1-\sqrt{\frac{\theta_{1}}{p_{23} k \bar{f}+\theta_{2}} \frac{\Delta R}{\bar{f}}}$. From IR-NM, $q_{2}<\frac{E^{L}[R]}{p_{23} \bar{f}}$. From Q1, $q_{2} \leq \frac{\bar{f}-\Delta R}{\bar{f}}$. Consequently, $q_{2}^{N M}$ depends on the magnitude relationship among $0, \bar{q}_{2}, \frac{E^{L}[R]}{p_{23} \bar{f}}, 1-\sqrt{\frac{\theta_{1}}{p_{23} \kappa \bar{f}+\theta_{2}}} \frac{\Delta R}{\bar{f}}$ and $\frac{\bar{f}-\Delta R}{\bar{f}}$. By examining all the forty patterns, we can derive $q_{2}^{N M}$ and $q_{1}^{N M}$.

In the case where IR-NM is binding, $f=\frac{E^{L}[R]}{p_{23} q_{2}}$. It is clear that $q_{1}=\frac{\Delta R}{\left(1-q_{2}\right) \bar{f}}$ satisfies IC-NM and maximizes the objective function. From Q2, $0 \leq q_{2} \leq 1$. The first order derivative of the objective function is $\Theta\left(q_{2}\right) \equiv$ $-\theta_{1} \frac{p_{23} \Delta R}{E^{L}[R]} \frac{1}{\left(1-q_{2}\right)^{2}}+\theta_{2}$ where $\frac{\partial \Theta}{\partial q_{2}^{2}}<0$. Therefore, the optimal solution of $q_{2}$ depends on which of the three conditions, that is, $0>\Theta(0)>\Theta\left(\bar{q}_{2}\right), \Theta(0)>0>\Theta\left(\bar{q}_{2}\right)$ or $\Theta(0)>\Theta\left(\bar{q}_{2}\right)>0$ is satisfied. From $\Theta\left(q_{2}\right)=0, q_{2}=1-\sqrt{\frac{\theta_{1}}{\theta_{2}} \frac{p_{23} \Delta R}{E^{L}[R]}}$. From P, $\frac{E^{L}[R]}{p_{23} \bar{f}}$. From Q1, $q_{2} \leq \frac{E^{L}[R]}{E^{H}[R]}$. Consequently, $q_{2}^{N M}$ depends on the magnitude relationship among $0, \bar{q}_{2}$, $1-$ $\sqrt{\frac{\theta_{1}}{\theta_{2}} \frac{p_{23} \Delta R}{E^{L}[R]}}, \frac{E^{L}[R]}{p_{23} \bar{f}}$ and $\frac{E^{L}[R]}{E^{H}[R]}$. By examining all the thirty patterns, we can derive $q_{2}^{N M}$ and $q_{1}^{N M}$.

By comparing the optimal solution when IR-NM is binding and that when IR-NM is not binding, we can derive the true optimal solution as shown in Figure 3 and Table 4.

\section{Process to Derive Figure 7}

We can easily confirm that $\left[u_{P}^{M}\left(q_{1}^{M}, q_{2}^{M}, f^{M}\right)-u_{P}^{N M}\left(q_{1}^{N M}, q_{2}^{N M}, f^{N M}\right)\right]$ is monotone increasing function of $\theta_{1}$ for any $\bar{f}$ and that $\lim _{\theta_{1} \rightarrow \infty} u_{P}^{N M}\left(q_{1}^{N M}, q_{2}^{N M}, f^{N M}\right)$ for any $\bar{f} \geq \Delta R$. Therefore, by checking the sign of $\left[u_{P}^{M}\left(q_{1}^{M}, q_{2}^{M}, f^{M}\right)-\right.$ $\left.u_{P}^{N M}\left(q_{1}^{N M}, q_{2}^{N M}, f^{N M}\right)\right]$ on $\bar{f}$ axis, we can know whether there is some area in which the prevention of the manipulation is optimal in the space of $\left(\theta_{1}, \bar{f}\right)$. By comparing $u_{P}^{M}\left(q_{1}^{M}, q_{2}^{M}, f^{M}\right)$ and $u_{P}^{N M}\left(q_{1}^{N M}, q_{2}^{N M}, f^{N M}\right)$, we can derive that it is optimal for the principal to allow the manipulation for any $\left(\theta_{1}, \bar{f}\right)$ iff $\Psi<\max \left\{-\bar{q}_{2} \theta_{2},-\frac{E^{L}[R]}{E^{H}[R]} \theta_{2}\right\}$.

The difference between $u_{P}^{M}\left(q_{1}^{M}, q_{2}^{M}, f^{M}\right)$ in region 4 of Figure 2 and $u_{P}^{N M}\left(q_{1}^{N M}, q_{2}^{N M}, f^{N M}\right)$ in region 4 and region 5 of Figure 4 is independent of $\bar{f}$. Therefore, the second pattern of Figure 7 realizes when one of the following two condition is satisfied: $u_{P}^{M}\left(q_{1}^{M}, q_{2}^{M}, f_{-}^{M}\right)>u_{P}^{N M}\left(q_{1}^{N M}, q_{2}^{N M}, f^{N M}\right)$ at the point where $\theta_{1}=\theta_{2} \frac{E^{L}[R]}{p_{23} \Delta R}\left(\frac{p_{23} \bar{f}-E^{L}[R]}{p_{23} \bar{f}}\right)^{2}$ and $\bar{f}=\frac{E^{H}[R]}{p_{23} \bar{q}_{2}}$ crosses or $\theta_{1}=\left[\Psi+\theta_{2} \frac{E^{L}[R]}{p_{23} \bar{f}}\right] \frac{p_{23} \bar{f}-E^{L}[R]}{p_{23} \Delta R}$, on which $u_{P}^{M}\left(q_{1}^{M}, q_{2}^{M}, f^{M}\right)$ in region 4 of Figure 2 becomes equal with $u_{P}^{N M}\left(q_{1}^{N M}, q_{2}^{N M}, f^{N M}\right)$ in region 6 of Figure 4 , crosses with $\theta_{1}=\theta_{2} \frac{E^{L}[R]}{p_{23} \Delta R}\left(\frac{p_{23} \bar{f}-E^{L}[R]}{p_{23} \bar{f}}\right)^{2}$ at the space $\bar{f} \geq \frac{E^{H}[R]}{p_{23} \bar{q}_{2}}$. From the former condition, $\Psi \leq-\theta_{2}\left\{\frac{E^{L}[R]}{E^{H}[R]} \bar{q}_{2}\right\}^{2}$ is derived. From the latter condition $-\theta_{2}\left\{\frac{E^{L}[R]}{E^{H}[R]} \bar{q}_{2}\right\}^{2} \leq \Psi \leq 0$ is derived. We have already confirmed that it is optimal for the principal to allow the manipulation for any $\left(\theta_{1}, \bar{f}\right)$ if $\Psi<$ 
$\max \left\{-\bar{q}_{2} \theta_{2},-\frac{E^{L}[R]}{E^{H}[R]} \theta_{2}\right\}$. Therefore, we can confirm that the second and the third pattern of Figure 7 appears respectively when $\max \left\{-\bar{q}_{2} \theta_{2},-\frac{E^{L}[R]}{E^{H}[R]} \theta_{2}\right\} \leq \Psi \leq 0$ and when $0<\Psi$.

\section{Ackowledgement}

I would like to thank Kei Fukuyama (Associate Professor at Tohoku University) for his great effort to hold the special workshop "Game Theoretic Analysis of Social Institutions" at Sendai. I would also like to thank the participants of the workshop and the anonymous referees for their comments to improve my research.

\section{REFERENCES}

[1] Che, Y. K., "Revolving doors and the optimal tolerance for agency collusion," RAND J. Econ., 26: 378-397 (1995).

[2] Fukumoto, J., and Tsuchiya, K., "A study on audit system of project evaluation," J. Infrastruct. Plan. Manage. (in Japanese), No. 772/IV-65: 79-95 (2004).

[3] Itoh, H., "Coalitions, incentives, and risk sharing," J. Econ. Theory, 60: 410-427 (1993).

[4] Kofman, F., and Lawarree, J., "Collusion in hierarchical agency," Econometrica, 61: 629-656 (1993).

[5] Kofman, F., and Lawarree, J., "On the optimality of allowing collusion," J. Public Econ., 61: 383-407 (1996).

[6] Laffont, J.-J., Incentives and Political Economy, Oxford University Press, Oxford (2000).

[7] Laffont, J.-J., and Martimort, D., “Collusion under asymmetric information," Econometrica, 65: 875-911 (1997).

[8] Laffont, J.-J., and Martimort, D., "Separation of regulators against collusive behavior," RAND J. Econ., 30: 232-262 (1999).

[9] Laffont, J.-J., and Tirole, J., A Theory of Incentives in Procurement and Regulation, MIT Press, Boston, MA (1993).

[10] Macho-Stadler, I., and Perez-Castrillo, J. D., An Introduction to the Economics of Information, Oxford University Press, Oxford (2001).

[11] Myerson, R., "Optimal coordination mechanisms in generalized principal-agent problems," J. Math. Econ., 10: 67-81 (1982).

[12] Niskanen, W. A., Jr., Bureaucracy and Public Economics, Edward Elgar (1994).

[13] Persson, T., and Tabellini, G., Political Economics: Explaining Economic Policy, MIT Press, Boston, MA (2000).

[14] Salanie, B., The Economics of Contracts, MIT Press, Boston, MA (1997).

[15] Strausz, R., "Collusion and renegotiation in a principal-supervisor-agent relationship," Scand. J. Econ., 99: 497-518 (1997).

[16] Tirole, J., "Hierarchies and bureaucracies: on the role of collusion in organizations," J. Law Econ. Organ., 2: 181-214 (1986).

[17] Tirole, J., "Collusion and the theory of organizations," In: Advances in Economics: Sixth World Congress, Vol. II, Laffont, J.J., Ed., Cambridge University Press, Cambridge (1992).

[18] Tirole, J., "The internal organization of government," Oxford Econ. Pap., 46: 1-29 (1994).

[19] Williamson, O., The Economic Institutions of Capitalism, Free Press (1985). 